${ }^{18}$ Leiden University Medical Center, Department of Rheumatology, Leiden, Netherlands; ${ }^{19}$ University Children's Hospital, University Medical Center Ljubljana, Department of Allergology, Rheumatology and Clinical Immunology, Ljubljana, Slovenia; ${ }^{20}$ University of Crete Medical School, Department of Rheumatology, Clinical Immunology and Allergy, Heraklion, Greece: ${ }^{21}$ Friedrich Alexander-University Erlangen-Nuremberg (FAU), Universitätsklinikum Erlangen, Department of Internal Medicine 3, Erlangen, Germany; ${ }^{22}$ University of Oxford, Nuffield Department of Orthopaedics, Rheumatology and Musculoskeletal Sciences, Oxford, United Kingdom

Background: A growing number of professional societies in clinical and medically related disciplines investigate evidence, make recommendations, and take action to advance gender equity. Evidence on women's advancement and leadership in the context of the European Alliance of Associations for Rheumatology, EULAR, is limited [1]

Objectives: The objective of the EULAR Task Force on Gender Equity in Academic Rheumatology was to establish the extent of the unmet need for support of female rheumatologists, health professionals and non-clinical scientists in academic rheumatology and develop a framework to address this through EULAR and Emerging EULAR Network (EMEUNET).

Methods: Potential interventions to accelerate gender-equitable career advancement in academic rheumatology were gathered from a narrative review of the relevant literature, expert opinion of a multi-disciplinary Task Force (comprised of 23 members from 11 countries), data from the surveys of EULAR scientific mem ber society leaders, EULAR and EMEUNET members, and EULAR Executive Committee members. These interventions were rated by Task Force members, who ranked each according to perceived priority on a five-point numeric scale from 1 = very low to 5 = very high.

Results: A framework of 29 potential interventions was formulated, which covers six thematic areas, namely, EULAR policies, advocacy and communication, EULAR Congress and associated symposia, training courses, mentoring/peer support, and EULAR funding (Figure 1).

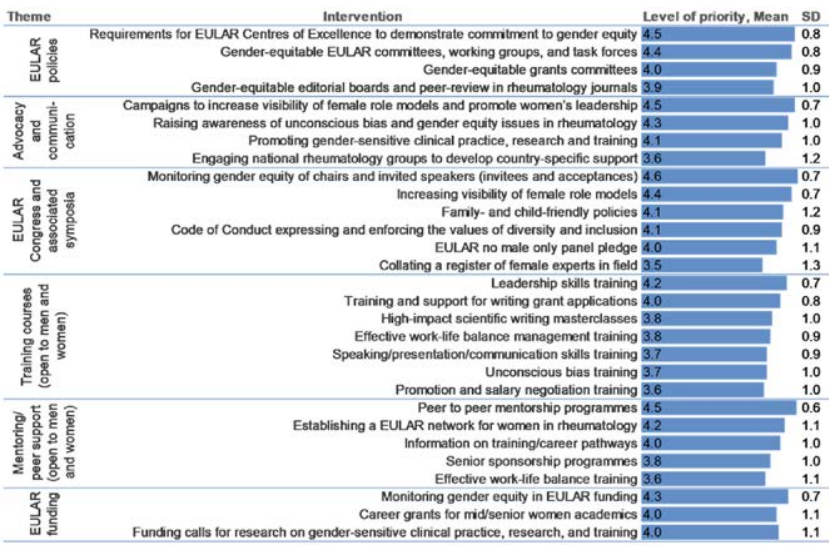

Figure 1. A framework of potential interventions with the levels of priority, mean and standard deviation (SD)

Conclusion: The framework provides structured interventions for accelerating gender-equitable career advancement in academic rheumatology. REFERENCES:

[1] Andreoli L, Ovseiko PV, Hassan N, et al. Gender equity in clinical practice, research and training: Where do we stand in rheumatology? Joint Bone Spine 2019;86(6):669-72

Acknowledgements: The task force is grateful to EULAR for funding this activity under project number EPI 024.

Disclosure of Interests: None declared

DOI: 10.1136/annrheumdis-2021-eular.1765

\section{OP0075 \\ EVALUATION OF A VIRTUAL REALITY TEACHING CONCEPT FOR MEDICAL STUDENTS DURING THE SARS-COV-2 PANDEMIC}

A. Pfeil ${ }^{1}$, F. Marcus ${ }^{2}$, T. Hoffmann ${ }^{1}$, P. Klemm ${ }^{3}$, P. Oelzner ${ }^{1}$, U. Müller Ladner $^{3}$, A. Hueber ${ }^{4}$, U. Lange ${ }^{3}$, G. Wolf ${ }^{1}$, G. Schett ${ }^{5,6}$, D. Simon ${ }^{5,6}$, A. Kleyer ${ }^{5,6}$. 'Jena University Hospital - Friedrich Schiller University Jena, Department of Internal Medicine III, Jena, Germany; ${ }^{2}$ Jena University Hospital - Friedrich Schiller University Jena, Department of Internal Medicine I, Jena, Germany; ${ }^{3}$ Campus Kerckhoff, Justus-Liebig University Gießen, Department of Rheumatology, Immunology, Osteology and
Physical Medicine, Bad Nauheim, Germany; ${ }^{4}$ Sozialstiftung Bamberg, Section Rheumatology, Bamberg, Germany; ${ }^{5}$ University Hospital Erlangen, Department of Internal Medicine 3, Rheumatology and Immunology, Erlangen, Germany; ${ }^{6}$ University Hospital Erlangen, German Center Immune Therapy, Erlangen, Germany

Background: The ongoing COVID-19 pandemic has disrupted face-to-face teaching of medical students and forced efforts in finding alternative approaches In order to help maintain high-quality education, a new virtual reality (VR)-based concept for training medical students in rheumatic and musculoskeletal diseases (RMD) has been developed. This VR training concept is based on the integration of real patient data with two- and three-dimensional visualized pathological joints from X-ray and computed tomography generated images.

Objectives: To evaluate the practicability and acceptance of the VR training application in the digital curricular education of medical students during the COVID-19 pandemic.

Methods: A short refresher lecture on rheumatic diseases (duration 60 minutes) was followed by presenting the VR training concept to the students. The VR training concept included the demonstration of three virtual patients with early rheumatoid arthritis, rheumatoid arthritis psoriatic arthritis regarding the symp toms, current medical problems, disease patterns at the imaging (conventional radiographs and high-resolution computed tomography) and therapy options. The practicability and acceptance of the VR was evaluated by medical students using a survey.

Results: The study encompassed 237 medical students (163 female, 73 male, one diverse, age range 20 to 40 years). $72 \%$ of the participants rated the virtual teaching as good or very good. $87 \%$ presented an expanded knowledge for rheumatoid arthritis and psoriatic arthritis through the VR. Moreover, $91 \%$ reported that the lecture provided a deeper understanding of RMD. Furthermore, $60 \%$ of the students asked for additional courses by VR.

Conclusion: The study highlighted the usefulness of innovative VR tools for teaching medical students digitally about RMD. VR applications can be a complementary educational modality for medical students, especially during the COVID-19 pandemic, to provide students with the best possible clinical experience while ensuring that patient, student, and staff safety is not compromised.

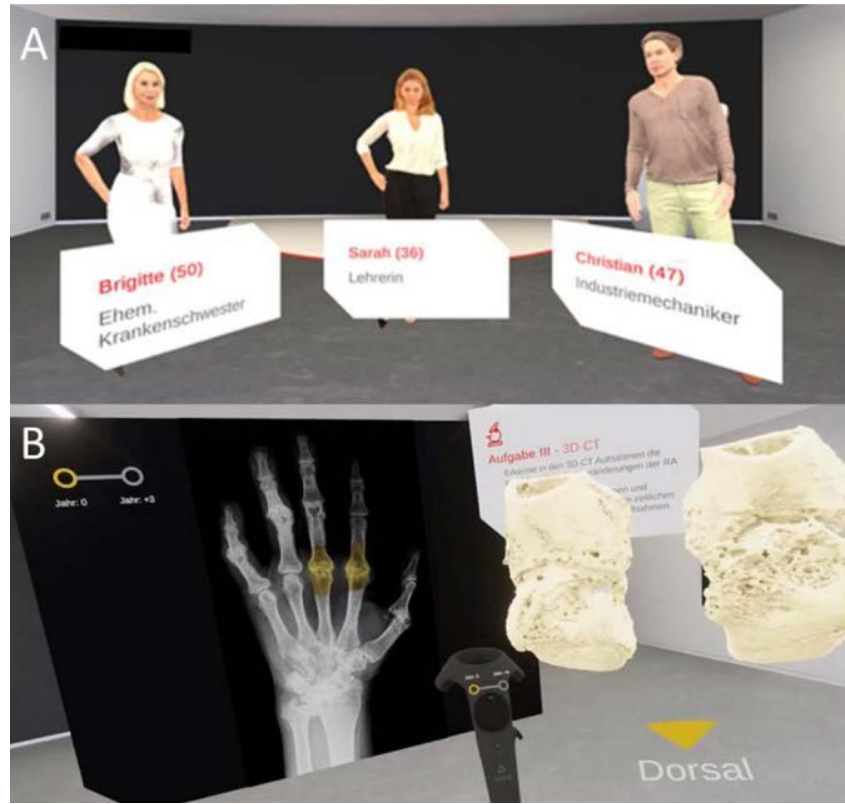

Figure 1. $A$ Screen view of virtual reality included three virtual patients with early rheumatoid arthritis (RA), RA, and psoriatic arthritis (PsA) and $\boldsymbol{B}$ demonstration of structural damage in RA and PsA using hand X-ray and high-resolution quantitative computed tomography images.

Disclosure of Interests: Alexander Pfeil Speakers bureau: Lilly Pharma Deutschland $\mathrm{GmbH}$, Franz Marcus: None declared, Tobias Hoffmann: None declared, Philipp Klemm Consultant of: Lilly Pharma Deutschland $\mathrm{GmbH}$ Peter Oelzner: None declared, Ulf Müller-Ladner Consultant of: Lilly Pharma Deutschland $\mathrm{GmbH}$, Axel Hueber Consultant of: Lilly Pharma Deutschland $\mathrm{GmbH}$, Uwe Lange: None declared, Gunter Wolf: None declared, Georg Schett: None declared, David Simon Consultant of: Lilly Pharma Deutschland GmbH, Arnd Kleyer Consultant of: Lilly Pharma Deutschland GmbH DOI: 10.1136/annrheumdis-2021-eular.2578 